\title{
THE INTERVERTEBRAL LIGAMENTS AS A SOURCE OF SEGMENTAL PAIN
}

\author{
D. C. Sinclair, W. H. Feindel, ${ }^{1}$ G. Weddell, and Murray A. Falconer ${ }^{2}$ \\ From the Department of Anatomy, University of Oxford, England
}

Steindler and Luck (1938) noted that localised lesions in the territory of the posterior primary divisions of the spinal nerves were capable of causing pain which radiated not only in this region but also in that of the anterior primary divisions. Kellgren (1939) and Lewis and Kellgren (1939) injected hypertonic saline in the mid-line of the back and caused pain of segmental distribution which they claimed was due to stimulation of the interspinous ligaments. In consequence, Kellgren $(1941,1942)$ stressed the importance of lesions of the posterior intervertebral ligaments as a cause of low back pain and sciatica. Indeed, he claimed (1941) that "in most patients suffering from so-called sciatica the pain is referred from a ligamentous or muscular lesion in the region of the hips or lumbosacral spine."

Study of the experimental work upon which this statement is based leads at once to certain criticisms of the technique employed, and of the inferences drawn. In the first place, the interspinous ligaments are mid-line structures connecting adjacent spinous processes. They are thin and membranous. Frazer (1940) states that they are "best developed in the lumbar region and only represented higher up by a fibrous or areolar web." It follows that accurate insertion of a needle into an interspinous ligament is difficult, and that to achieve it the point of the needle must lie exactly in the mid-line. Moreover, in any situation other than the lumbar region, there can be no guarantee that the injected fluid will be confined within the ligament. Kellgren (1939) did not even attempt to inject fluid in the mid-line. He stated that the injecting needle was introduced "exactly in the mid-line and passed through the supraspinous ligament. It is then moved slightly to one side until the tough interspinous ligament is felt with the needle point. At this moment the subject feels a unilateral pain, and the saline is injected." Similarly, Lewis and Kellgren state that the needle is inserted until " at a depth of about 1 to $3 \mathrm{~cm}$., the deep ligament is encountered. Carrying the needle a half-centimetre to one side of the mid-line usually gives a little preliminary pain that is felt unilaterally. The injection of $0 \cdot 3$ c.c. of 6 per cent. saline (or less) is made at once ..." In no case was the position of the needle point ascertained radiographically. It is unlikely therefore that the interspinous ligaments were the only, or indeed the principal, structures stimulated by these procedures, and before attributing pain to this cause it is reasonable that other sources should be excluded. Lewis and Kellgren admit the possibility of other structures being stimulated when they say that "the stimulus is ... applied to a small ligament or to muscle fibres attached to it." Furthermore it must be remembered that when a dyed solution was used instead of saline, Kellgren (1938) found that after injecting $0 \cdot 3$ cubic centimetre into muscle the "portion of muscle coloured by the dye was about $2 \mathrm{~cm} . \times 3 \mathrm{~cm} . \times 1 \mathrm{~cm}$. ..."

It is therefore misleading to insist, as do Lewis and Kellgren, that the stimulus is "quite local." Fig. 1 is a diagram to scale, showing the relationships in a cross-section through a thoracic vertebra. The line $A B$ represents possible situations of the point of the needle, and the shading shows the area into which the saline would be dispersed, taking as a basis the one centimetre which was the shortest diameter of the volume stained after injecting $0 \cdot 3$ cubic centimetre of methylene blue solution (Kellgren 1938). Within this shaded area lies part of the course of the medial branch of the posterior primary division of the spinal nerve, and the possibility of direct stimulation of this nerve must be considered. Lewis and Kellgren

\footnotetext{
1 Rhodes Scholar, Nova Scotia, and Merton College, Oxford.

${ }^{2}$ I.ecturer in Neurosurgery, Otago Lniversity, New Zealand.
} 
refer to preliminary pain caused by carrying the print of the needle laterally, and this could be explained readily by direct nerve contact. Johnston (1908) showed that in the thoracic region these branches "pass downwards, inwards and backwards superficial to the multifidus spinae muscle, until they reach the spinous processes of the upper thoracic vertebrae. Before becoming superficial they descend by the sides of the spinous processes a distance which gets gradually greater as we pass from the first to the lowest member of the series, the nerves increasing in length." (Fig. 2.) In the thoracic region, therefore, injection near the mid-line would be almost certain to affect these nerves. Lower down, however, in the lumbar region, they " run close to the articular processes of the vertebrae and end in the multifidus " (Gray's Anatomy 1946). If, then, the results are due to stimulation of the medial branches of the posterior primary divisions of spinal nerves, injection of hypertonic saline near the midl-line

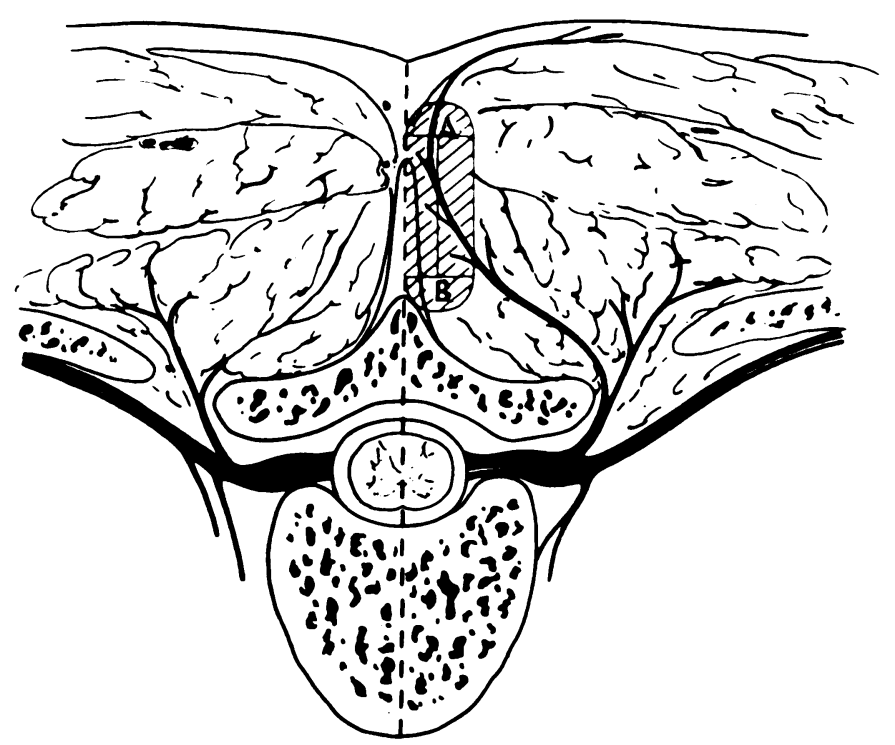

IIG. 1

Diagram of transverse section through a thoracic vertebra. (adapted from Spalteholz). If the technique of Lewis and Kellgren is followed, the portion of the medial branch of the posterior primary division of the spinal nerve lying within the cross-hatched area is liable to be directly affected by the injected saline.

should elicit pain more readily in the thoracic region than in the lumbar region. On the other hand, if the results are due to stimulation of interspinous ligaments, the reverse should hold good, owing to the larger size of these structures in the lumbar region.

But, since neither Kellgren (1939) nor Lewis and Kellgren (1939) made accurate measurements of the position of the point of the needle, there remains another possibility. It may be that the "tough interspinous ligament", or the "deep ligament" which they encountered to one side of the mid-line, was in fact the ligamentum flavum. If this is so, there is a distinct chance that in some cases the injected saline affected the posterior nerve root itself, since Falconer, Glasgow, and Cole (1947) demonstrated that extrathecal nerve roots in the lumbar region can be injected by means of a needle inserted about half an inch from the mid-line and directed straight forwards through the ligamentum flavum.

These facts cast doubt on the validity of Lewis and Kellgren's assumption that their results were due simply to stimulation of the interspinous ligaments. 


\section{EXPERIMENTAL STUDY}

In a preliminary study of the problem, 6 per cent. saline was injected in various sites and at various depths in the lumbar and thoracic regions of the backs of a number of volunteers. The injections were made on the $\mathrm{X}$-ray table, the needle being inserted through a small intradermal button of 1 per cent. novocaine. The maximum quantity of saline injected in any one situation was usually 0.3 cubic centimetre, in conformity with the procedure of Lewis and Kellgren; but in some instances $0 \cdot 6$ cubic centimetre was injected. Before injection, the point of the needle was moved slightly in the tissues both vertically and horizontally, and the sensations were noted. The excursion of the point was not actually measured, but it must have been small because in all cases the point lay at a depth of 1 centimetre or more, and it was not withdrawn. In other experiments no injection was made, but the point of the needle was used as a probe, being partly withdrawn and reinserted at intervals in order to search a considerable area thoroughly. The position of the point at any desired moment was determined by taking one lateral radiograph, and two antero-posterior radiographs, with a tube shift of 10 centimetres, in order to give adequate parallax. In some instances fluoroscopic screening was used.

Observations - The importance of radiographic control was soon established, because without its aid it was found very difficult to predict with accuracy the position of the needle point. Indeed in every case it was found necessary to confirm the position of the needle point before injection, instead of simply using radiography as a check. The technique was timeconsuming, but the position of the needle was never in doubt. In most cases, passage of the needle through deep tissues was not accompanied by discomfort other than a sense of vague " pushing," but in a few instances dull, unpleasant, aching pain was felt locally near the mid-line. On each such occasion radiographs showed that the point of the needle was in contact with the vertebra above or below, and this pain may be attributed therefore to stimulation of the periosteum by the needle point.

It was found that intervertebral injection of $0 \cdot 3$ cubic centimetre of 6 per cent. saline made in the lower thoracic region, with the needle point at depths varying up to 3 centimetres and as near the mid-line as possible, always caused pain which was felt locally, and was also referred to the distribution of the spinal segment. It was usually felt on one side of the body

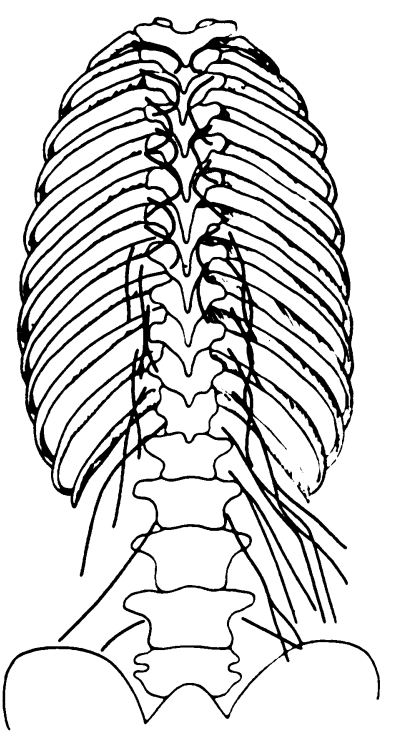

IIG. 2

l)iagram showing the deep course (in one subject) of those branches of the posterior primary divisions of the spinal nerves which eventually became cutaneous. Contrast their position, relative to the mid-line, in the thoracic and in the lumbar regions. Note that muscular branches are not indicated in the drawing (after Johnston).

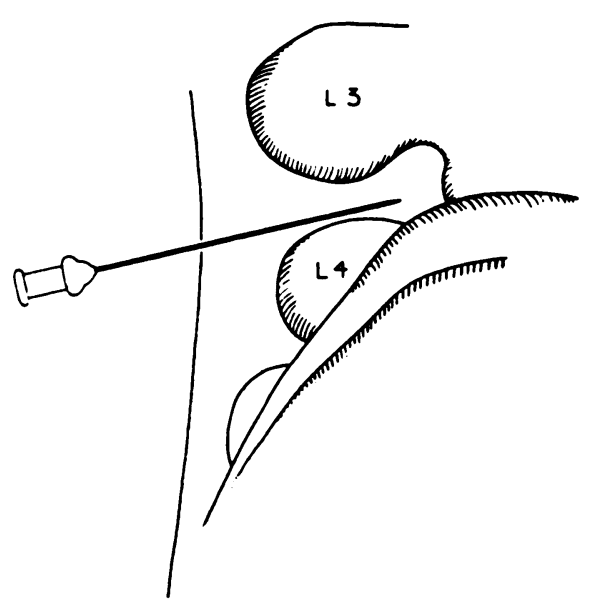

FIG. 3

Tracing from control radiograph of midline injection in the lumbar region, indicating the position of the needle point in the interspinous ligament.

vol. $30 \mathrm{~B}$, No. 3 , AUgust 1948 
only, though occasionally local pain transgressed the mid-line and was felt on both sides. If the needle was accurately placed in the mid-line the intensity and duration of the resultant pain were small; but if the point deviated even a few millimetres to one side, the pain, though similar in distribution, was much more intense; it lasted longer and it was accompanied by referred deep tenderness and cutaneous hyperalgesia. In fact it corresponded closely to the description given by Kellgren (1939). With the point of the needle half a centimetre from the mid-line, an injection of 0.1 cubic centimetre at a depth of $\mathbf{2}$ centimetres was sufficient to produce well-marked referred pain lasting for five minutes, deep tenderness lasting fifteen minutes, and cutaneous hyperalgesia persisting for ninety minutes. It should perhaps be noted that an injection of sodium penicillin $(20,000$ units per cubic centimetre in distilled water) made into the same site, later reproduced the whole clinical picture except that it was of brief duration.

In the lumbar region, where the interspinous ligaments are thicker and more readily injected, it was found that injection of $0 \cdot 3$ cubic centimetre of saline exactly in the mid-line gave rise only to mild local discomfort, and sometimes to no subjective sensation at all. An injection of 0.6 cubic centimetre was required before much pain was produced; even then, though of characteristically unpleasant quality, it was confined to a local area and was not referred segmentally. In one case a series of stimulations was carried out between the third and fourth lumbar vertebrae, each stimulation by the point of the needle being progressively more lateral. Not until the needle point was 3 centimetres from the mid-line, at a depth of 2 centimetres from the skin, was there referred pain, and an injection carried out at this point gave results similar to those described for the thoracic region.

It should be mentioned that, whereas in thoracic injections there was little or no sense of resistance, lumbar mid-line injections were accompanied by much resistance, suggesting that the fluid was being injected into dense tissue. Furthermore, local pain produced by injections in the mid-line in this region tended to persist; in one subject after an injection of 0.6 cubic centimetre there was considerable disability for a period of twenty-four hours. During this time there was never radiation of pain; it remained confined to the original site, around and just distal to the needle, and it retained its unpleasant quality. The persistence of the pain may be attributed to disruption of the ligament by the injected fluid, and it is interesting to note that an injection of novocaine into the site was temporarily successful in abolishing the pain.

Summary of experimental observations-To summarise it may be stated that in the thoracic region, injections made as nearly as possible in the mid-line caused referred pain much less readily than injections to one side of the mid-line. In the lumbar region injection of as much as $\mathbf{0} \cdot \mathbf{6}$ cubic centimetre of hypertonic saline into the interspinous ligament in the mid-line failed to cause referred pain; it caused only local pain in the back. Only when the needle point was carried laterally to a distance of about 3 centimetres was referred pain caused in one case by injecting $0 \cdot 3$ cubic centimetre of saline at a depth of 2 centimetres. It may therefore be said that in this series of experiments the results were consistent with the hypothesis that referred pain is caused by direct stimulation of nerve trunks, and inconsistent with the view that it is due to the stimulation of interspinous ligaments.

\section{DISCUSSION}

Our results do not support the contention of Lewis and Kellgren that stimulation of an interspinous ligament by injection of hypertonic saline is capable of causing referred pain in the distribution of the spinal segment innervating the ligament. The results of injecting hypertonic saline close to the mid-line in the thoracic and lumbar regions cannot be explained on this basis. Furthermore, repeated failure to produce segmental pain by the injection of relatively large quantities of saline exactly in the mid-line of the lumbar region constitutes evidence against the theory. On the other hand, the findings are explained easily if it is assumed that they are due to direct stimulation of a nerve trunk in the vicinity. 
Lewis and Kellgren observe that " there is no possibility of direct stimulation of spinal nerves," but they give no support for this statement other than that the stimulus is "local," and that "the response is invariable and immediate." It has been pointed out that the first of these reasons is misleading, owing to the relatively wide spread of saline after injection into a muscle; and the second could be used with equal force to sustain the opposite contention. Kellgren (1938) tries to distinguish the subjective results of stimulating nerve trunks and muscle by observing that when hypertonic saline is injected into a muscle and gives rise to a sensation of burning pain and pins and needles in the cutaneous distribution of a nerve trunk, this sensation is "strikingly different from that produced by saline injected into muscle": he attributes such pain to the stimulation of a nerve. Nevertheless, both sensations are, in fact, the consequence of injecting saline into a muscle, and both are presumably conducted to consciousness by stimulation of sensory nerves in the muscle. In effect, therefore, both types of pain have a similar etiology, the difference being one of degree, and the more unusual type of pain is perhaps attributable to the injection of hypertonic saline directly into the substance of a mixed nerve trunk rather than into the immediate vicinity of the nerve. In this investigation there appeared to be only one type of pain, but it varied greatly in intensity according to the exact position of the needle. This finding may be interpreted as being due to the varying distances from the nerve trunk at which injections were made. In short, there appears to be no valid evidence against the assumption that pain produced by injecting hypertonic saline near the mid-line of the back is due to direct stimulation of one or more of the local nerve trunks.

Kellgren (1942) states that "the supraspinous ligament is intensely sensitive; the structures surrounding the neural arch are somewhat less so, and the vertebral bodies and discs are relatively insensitive." The first two parts of this contention appear to be based on the results of injecting hypertonic saline; for this reason they are open to question. No evidence is offered in support of the third part. Experience of lumbar puncture does not suggest that the supraspinous ligament is "intensely sensitive" to types of stimulation other than chemical irritation. In this series of experiments, piercing or scratching the supraspinous ligament with the point of a needle caused negligible pain.

Kellgren (1942) also maintains that stimulation of the supraspinous ligament causes local pain; while the deep-lying structures surrounding the vertebrae give rise to segmental pain; and the intermediate muscle mass gives pain of " modified segmental distribution." Elsewhere (1939) he remarks that the degree to which pain is localised depends on the depth from the body surface of the tissue under investigation: superficial structures give rise to local pain; deeper structures (in the chest wall) "give rise to diffuse pain of segmental distribution whether the structure stimulated be muscle, ligament or periosteum." In this statement there appears to be confusion between the ability to localise painful stimuli and the occurrence of referred pain. If these two factors are separated, the findings admit of a more simple explanation. It is now assumed generally that accuracy of stimulus-localisation in the skin is a function of the density of innervation (Weddell 1945). There is no reason to suppose that it is otherwise in deeper tissues, and since the density of innervation of these structures is far below that of the skin, it is not surprising that localisation of painful stimuli should be less accurate (Feindel, Weddell, and Sinclair 1948). The relative accuracy with which pain is localised after injection of hypertonic saline into the supraspinous ligaments is probably explained by leakage of fluid into the more densely innervated subcutaneous tissue. On the other hand these ligaments are superficial, and even without radiographic control it is easy to make certain that most of the saline remains in the vicinity of the ligament itself, and that it does not penetrate far enough to stimulate the medial branch of the posterior primary division. The absence of reference of pain from the supraspinous ligaments may thus be explained. In the case of muscles, it has already been submitted that pain produced by the injection of saline is the result of stimulation of the nerve trunks. Johnston (1908)

vol. $30 \mathrm{~B}$, No. 3 , AUgust 1948 
showed that the medial branches of the posterior primary divisions often communicate freely with their immediate neighbours in a tortuous course through deep tissues (Fig. 4). It is therefore unlikely that stimulation of this network would alwavs produce pain in a clear-cut segmental pattern. This is probably the reason for the "modified segmental distribution " described by Kellgren. Finally, it is likely that injections made round accessible parts of the vertebrae will be poorly localised if they do not stimulate a nerve trunk. They are, however, capable of impinging upon the posterior nerve root itself, with the production of full segmental pain.

Elliott (1944) using Kellgren's technique, found much variation in the site of reference of pain from the injection of hypertonic saline into the fourth and fifth lumbar "interspinous ligaments " in different subjects. It is interesting also to note that Gray (1947) denied that pain produced by this technique was referred on a segmental basis.

Kellgren (1942) argued that disease of the vertebral bodies seldom produced pain unless the neural arches were also involved, and (1941) suggested that localised kyphosis due to disruption of the nucleus pulposus might cause a variety of clinical syndromes. "The stretched supraspinous ligament may become painful, producing local pain in the mid-line of the back together with slight limitation of flexion and extension of the spine: then the

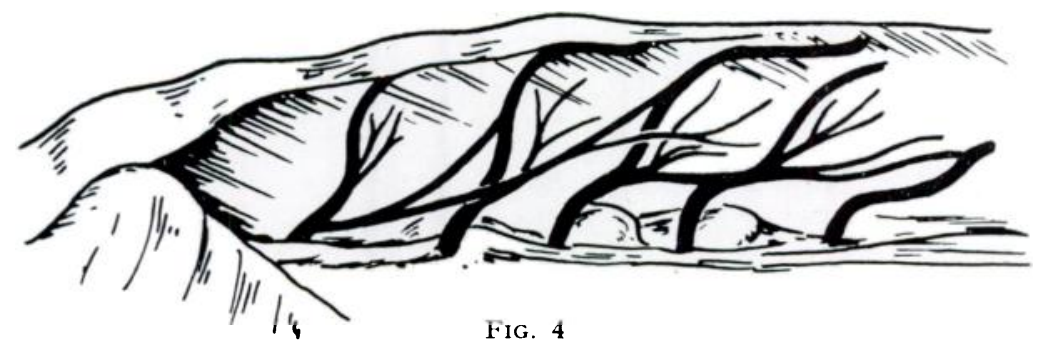

Lateral view of a dissection showing free communication between the medial branches of the posterior primary divisions arising from different spinal nerves in the thoracic region (after Johnston).

interspinous ligament and ligamentum flavum may become painful, giving rise to deep pain of segmental distribution together with great limitation of extension of the spine." There is nothing by which to prove that this hypothesis is untrue, but on anatomical grounds it is not well founded. Local lesions in the mid-line of the back may certainly cause profound disturbance $-\mathrm{a}$ fact of which both qualified and unqualified manipulative surgeons have long been aware. Steindler and Luck noted that they were able to suppress referred pain from such lesions by the injection of a local anaesthetic into the painful points. Kellgren (1941) confirmed this finding in sciatic pain, but he noted that it was unusual for the signs and symptoms to be suppressed completely by this procedure, explaining his failure by the difficulty in securing complete anaesthesia of the deep-lying structures. It is submitted, however, that the reason for incomplete suppression resides not in this difficulty, but in the failure to block local nerve trunks. In short, the evidence at present indicates that these lesions produce referred pain (which may simulate visceral disease) by direct involvement of some part of the segmental sensory nervous pathway.

\section{SUMMARY AND CONGLUSIONS}

1. The work of Kellgren (1939) and Lewis and Kellgren (1939) on the production of segmental pain by stimulation of structures near the mid-line of the back has been reviewed, and a number of experiments have been carried out with the object of testing the validity of their conclusions. 
2. On both anatomical and experimental grounds there is reason to believe that pain produced in this manner does not arise, as was assumed by Lewis and Kellgren, from irritation of the interspinous ligaments, but is rather due to the stimulation of nerve trunks in the vicinity. The views of Kellgren $(\mathbf{1 9 4 1}, \mathbf{1 9 4 2})$ on the etiology of pain in certain cases of sciatica, and on the part played by intervertebral ligaments in the production of pain in the back, have been discussed. They have been shown to rest on an inadequate foundation.

3. The importance in investigations of this kind of a detailed anatomical survey has been emphasized.

Our thanks are due for the invaluable assistance and interest of Dr A. D. Kemp and Miss Carmill, of the Radiological Department of the Radcliffe Infirmary, Oxford. We would also record our appreciation of the great help rendered by the volunteers who participated in this investigation. The expenses of these investigations were defrayed by a grant to one of us (G. W.) by the Medical Research Council of Great Britain which is gratefully acknowledged.

\section{REFERENCES}

Elliott, F. A. (1944): Tender Muscles in Sciatica. Lancet, 1, 47.

Falconer, M. A., Glasgow, G. L., and Cole, D. S. (1947): Sensory Disturbances occurring in Sciatica due to Intervertebral Disc Protrusions; Some Observations on the Fifth Lumbar and First Sacral Dermatomes. Journal of Neurology, Neurosurgery, and Psychiatry, N.S. 10, 72.

Feindel, W. H., Weddell, G., and Sinclair, D. C. (1948): Pain Sensibility in Deep Somatic Structures. Journal of Neurology, Neurosurgery, and Psychiatry, N.S. 11, 113.

Frazer, J. E. (1940): The Anatomy of the Human Skeleton. Churchill, London, Edition 4.

Gray's ANatomy (1946): Longmans Green \& Co., London, Edition 29.

Gray, C. (1947): The Causes and Treatment of Sciatic Pain. International Abstracts of Surgery, 85, 417. Johnston, H. M. (1908): The Cutaneous Branches of the Posterior Primary Divisions of the Spinal Nerves and their Distribution in the Skin. Journal of Anatomy and Physiology, 43, 80.

Kellgren, J. H. (1938): Observations on Referrẹd Pain arising from Muscle. Clinical Science, 3, 175.

Kellgren, J. H. (1939): On the Distribution of Pain arising from Deep Somatic Structures with Charts of Segmental Pain Areas. Clinical Science, 4, 35.

Kellgren, J. H. (1941): Sciatica. Lancet, 1, 561.

Kellgren, J. H. (1941-42): Conditions of the Back simulating Visceral Disease. Proceedings of the Royal Society of Medicine (Section of Orthopaedics), 35, 199.

Lewis, T., and Kellgren, J. H. (1939): Observations relating to Referred Pain, Viscero-Motor Reflexes, and other Associated Phenomena. Clinical Science, 4, 47.

Steindler, A., and Luck, J. V. (1938): Differential Diagnosis of Pain low in the Back. Journal of the American Medical Association, 110, 106.

Weddell, G. (1945): The Anatomy of Cutaneous Sensibility. British Medical Bulletin, 3, 167.

vol. $30 \mathrm{~B}$, No. 3 , AUGUST 1948 